\title{
Development of the PARMS marker of the TAC1 gene and its utilization in rice plant architecture breeding
}

\author{
Ju Gao • Haifu Liang • Juan Huang • Dongjin Qing • Hao Wu • \\ Weiyong Zhou • Weiwei Chen · Yinghua Pan • Gaoxing Dai • Lijun Gao • \\ Guofu Deng $(\mathbb{D}$
}

Received: 31 May 2020/ Accepted: 9 December 2020/Published online: 26 February 2021

(C) The Author(s) 2021

\begin{abstract}
The ideal plant architecture is a new strategy for super high yield breeding of rice. Tiller angle is an important plant architecture character of rice. A reasonable tiller angle is a key factor for the ideal plant architecture and achieving high-yield breeding. Molecular design breeding is the most potential new direction of crop breeding in the future. The development of accurate and efficient functional
\end{abstract}

Ju Gao, Haifu Liang have contributed equally to this work

Supplementary Information The online version contains supplementary material available at (https://doi.org/10.1007/ s10681-020-02747-y).

J. Gao · J. Huang $\cdot$ D. Qing $\cdot$ H. Wu $\cdot$ L. Gao $(\bowtie)$

Guangxi Crop Genetic Improvement and Biotechnology

Laboratory, Guangxi Academy of Agricultural Sciences,

Nanning, China

e-mail: gaolijun@gxaas.net

J. Gao

e-mail: gaojufly@126.com

J. Huang

e-mail: huangjuan@gxaas.net

D. Qing

e-mail: 64315946@qq.com

H. Wu

e-mail: 287642852@qq.com

H. Liang · W. Zhou - W. Chen · Y. Pan · G. Dai

Rice Research Institute, Guangxi Academy of

Agricultural Sciences, Nanning, China

e-mail: lianghaifu7711@gxaas.net molecular markers of target trait genes is crucial for molecular design breeding. The TAC1 (Tiller Angle Controlling) gene is the primary gene that regulates tiller angle in rice. This gene can be used to improve the compact plant architecture of indica and japonica rice varieties. The SNP variation from A to $\mathrm{G}$ at the fourth intron $3^{\prime}$ splicing point in TACl changes plant architecture. Based on the SNP variation, PM-TAC1 was successfully developed as a fluorescent functional molecular marker, via the penta-primer amplification refractory mutation system. Ninety-three rice materials were genotyped using this marker, and the marker was effectively used in rice plant architecture breeding. The

\footnotetext{
W. Zhou

e-mail: zhouweiyong@gxaas.net

W. Chen

e-mail: chenwei0078@163.com

Y. Pan

e-mail: panyinghua2008@163.com

G. Dai

e-mail: 25266220@qq.com

G. Deng $(\bowtie)$

Guangxi Academy of Agricultural Science,

Nanning 530007, China

e-mail: dengguofu163@163.com
} 
successful development of this marker will contribute to the molecular breeding of rice plant architecture.

\section{Keywords $T A C 1 \cdot$ Plant architecture - PARMS marker $\cdot$ Rice $\cdot$ MAS breeding}

\section{Introduction}

New plant architecture breeding (or super rice breeding) of rice was proposed in the 1990s (Khush 1996). Super hybrid rice varieties with the ideal plant architectures are essential to increase global food supply and ensure food security. Tillering angle is one of the indexes used in measuring the degree of plant tightness. It is an important plant architecture character of rice. It has a considerable influence on canopy structure, photosynthetic efficiency, material production, and disease resistance of rice. (Wang and $\mathrm{Li}$ 2005; Sun et al. 2019).

Although tillering angle is affected by the environment, it is primarily determined by genetic factors (Dong et al. 2016). The TACl (Tiller Angle Controlling 1) is a major quantitative trait locus of tiller angle in rice ( $\mathrm{Yu}$ et al. 2007). TAC3, together with $T A C l$ and $D 2$, greatly controls tiller angle in rice cultivars (Dong et al. 2016). The gene PROGl controls the creeping growth habit of wild rice, which is essential to reveal the molecular mechanism of rice evolution (Jin et al. 2008; Tan et al. 2008). LPAl is a loose plant architecture gene that controls tillering angle and leaf angle by controlling the growth of the paraxial surface between the tiller and the leaf angle (Wu et al. 2013; Liu et al. 2016). Spk(t) controls the tillering angle of rice, and the dominant allele from Kasalath increases tillering angle that makes rice present scattered characteristics (Yamamoto T 1997; Miyata et al. 2005). TIG1 positively regulates EXPA3, EXPB5, and SAUR39 expression to promote cell elongation and increase tiller angle (Zhang et al. 2019a, b). The rice phytochrome-interacting factorlike protein OsPIL15 regulates tiller angle through light and gravity signals in rice (Xie et al. 2019). Numerous genes have been demonstrated to affect tillering angle through auxin regulation. $L A Z Y 1$ affects tillering angle by negatively regulating the polar transport of auxin. After the loss of $L A Z Y 1$ function, the polar transport capacity of auxin is greatly enhanced, thereby enhancing stem geotropism and making the whole plant loose
(Li et al. 2007). OsBRXL4 regulates the centripetal and tillering angle of rice by affecting the nuclear localization of LAZY1 (Li et al. 2019). OsPIN2 depends on the auxin regulatory pathway to participate in the regulation of rice plant architecture. OSPIN2 overexpression in rice inhibits $O S L A Z Y 1$ expression, thereby increasing tiller and tiller angle and decreasing plant height (Chen et al. 2012). OsLIC1 regulates the plant architecture characteristics of rice, such as leaf angle, plant height, tiller angle, and grain number per ear, through the BR signaling pathway (Wang et al. 2008). OsLIC1 is a direct target of $O s B Z R 1$, which can be phosphorylated by GSK3 kinase. As a zinc finger transcription factor antagonistic to BZRl, it weakens the BR signal (Zhang et al. 2012). OsmiR167a represses its targets, namely, OsARF12, OsARF17 and OsARF25, to control rice tiller angle by fine-tuning auxin asymmetric distribution in shoots (Li et al. 2020).

The tiller angle genes that control the two extreme types of rice, namely, dispersed or erect, such as PROG1, LPA1, Spk(t) and TIG1, have little practical breeding value. By contrast, the major QTL TAC1 increases the tillering angle of rice lines appropriately (Yu et al. 2007; Jiang et al. 2012), and thus plays an important role in breeding. TACl was isolated via mapbased cloning by using the loose plant architecture indica rice variety 'IR24' as the genetic background and the compact infiltrating line 'IL55' that infiltrates a few chromosome segments of the japonica rice variety 'asominori' as the material. (Yu et al. 2007; Jiang et al. 2012). A comparison of the gene sequences of TAC1 from IR24 and IL55 revealed an important SNP variation from $A$ to $G$ at the $3^{\prime}$ splicing site of the fourth intron. The genome sequence changed from "AGGA" in TACl to "GGGA" in tacl. As a result, the intron could not be cut normally during mRNA processing, and poly (A) was added ahead of time, resulting in the cDNA of TACl and tacl to encode the same amino acid sequence but with completely different $3^{\prime}$ untranslated regions. TACl with "AGGA" is a loose plant architecture, whereas tacl with "GGGA" is a compact plant architecture (Yu et al. 2007).

The penta-primer amplification refractory mutation system (PARMS) is an SNP genotyping technology based on the tetra-primer amplification refractory mutation system (Ye et al. 2001). In this technique, five primers (a pair of universal fluorescent primers, a pair of allele-specific primers, and a pair of reverse shared primers) are used to amplify SNP or short indel 
loci with allele specificity. Genotyping is then conducted by fluorescence scanning (Zhang et al. 2019a, b; Lu et al. 2020).

Molecular marker-assisted selection (MAS) is a highly efficient breeding method because it offers a rapid and precise selection of the desired genes. These markers are based on PCR and gel electrophoresis. Herein, on the basis of SNP variations in the TAC1 gene from $A$ to $G$ at the $3^{\prime}$ splicing site of the fourth intron, we developed the functional molecular marker PM-TAC1 via PARMS and utilized it in MAS breeding to improve the plant architecture of rice.

\section{Materials and methods}

\section{Experimental materials}

Ninety-three rice experimental materials were planted in the experimental field of Guangxi Academy of Agricultural Sciences. These materials included 31 maintainer lines of CMS lines, 6 temperature-/photoperiod-sensitive genic male sterile (TGMS) lines, 24 restorer lines of CMS lines, and 32 varieties (Table S1). Plant architecture was investigated at the heading stage.

\section{Primer design of the PARMS markers of TACI}

BLAST was used for sequence alignment, and the online primer design tool Primer3Plus (https:// primer3plus.com) was utilized to design the primer sets for all markers. The PARMS molecular marker PM-TAC1 was designed based on the SNP variations from $\mathrm{A}$ to $\mathrm{G}$ at the $3^{\prime}$ splicing site of TACl intron 4 . These variations cause the plant architecture to change from loose to compact. The marker consists of three specific primers of TAC1. Two different bases of the SNP variations were introduced into the designed forward primers. In addition, two common primers that are consistent with the underlined parts of the two forward primers had different fluorescent marks at the tail. The underlined sequence was annealed to the oligonucleotides labeled with the FAM/HEX fluorophore (Table 1.). Using this technique, three primers were designed according to the sequence of $T A C 1$, and two universal fluorescent primers were added to the PCR reaction system for amplification (Fig. S1). According to the SNP variations, the allele sequence of TACl was amplified to obtain the FAM fluorescence signal value by matching with the forward primer TAC1-Fg and the HEX fluorescence signal value and by matching with the forward primer TAC1-Fa. When the rice sample was heterozygous at this site, two forward primers were simultaneously amplified.

\section{Sample collection and DNA extraction}

The leaves of the 93 rice materials were used for DNA extraction via the CTAB method (Murray and Thompson 1980). The extracted DNA was dissolved in $1 \times$ TE buffer for subsequent PCR amplification.

PCR amplification and genotyping assays

The TACl gene of these rice varieties was amplified via PCR. The master mix for the PARMS markers was purchased from Gentides Biotech Co., Ltd. (Wuhan, China). The PCR reaction system $(10 \mu \mathrm{L})$ included 5 $\mu \mathrm{L} 2 \times$ PARMS master mix (containing two common fluorescent primers, PCR buffer, dNTP and Taq enzymes, and internal standard ROX), $0.15 \mu \mathrm{L}$ $10 \mathrm{mM}$ TAC1-Fg primer, $0.15 \mu \mathrm{L} 10 \mathrm{mM}$ TAC1-Fa primer, $0.4 \mu \mathrm{L} 10 \mathrm{mM}$ TAC1-R primer, $1 \mu \mathrm{L} 50 \mathrm{ng}$ DNA template, and $3.3 \mu \mathrm{L}$ of $\mathrm{ddH}_{2} \mathrm{O}$. Touchdown PCR was performed as follows: $95^{\circ} \mathrm{C}$ for $5 \mathrm{~min}$, followed by 10 cycles of $95{ }^{\circ} \mathrm{C}$ for $20 \mathrm{~s}$ and $65^{\circ} \mathrm{C} \mathrm{(-}$ $0.8^{\circ} \mathrm{C}$ per cycle) for $1 \mathrm{~min}$, and 32 cycles of $95{ }^{\circ} \mathrm{C}$ for $20 \mathrm{~s}$ and $57{ }^{\circ} \mathrm{C}$ for $1 \mathrm{~min}$ and $72^{\circ} \mathrm{C}$ for $7 \mathrm{~min}$.

The PCR products were analyzed on a Multiscan Spectrum (Tecan Infinite 200-TWT, Grodig, Austria), which can detect the FAM, HEX, and ROX fluorescent signals. The fluorescence signal value was then filed by the SNP decoder software (http://www.snpway. com/snpdecoder01/) to obtain each sample amplification of the FAM and HEX fluorescence signal strengths. Each point with the graphical output signal was acquired according to the fluorescence signal intensity. Automated genotyping was performed to generate genotype results. According to the analysis of fluorescence signal values, the fluorescence signal of FAM (blue) obtained via fluorescence scanning was the tacl allele $(\mathrm{G})$ type material of compact plant architecture, whereas the fluorescence signal of HEX (green) was the TAC1 allele (A) type material of loose plant architecture. The fluorescence scanning result was red, indicating that the sample was heterozygous. 
Table 1 PARMS primer sets for genotyping

\begin{tabular}{lll}
\hline $\begin{array}{l}\text { PARMS } \\
\text { primer }\end{array}$ & Sequences & Remarks \\
\hline $\begin{array}{l}\text { TAC1-Fg } \\
\text { TAC1-Fa }\end{array}$ & GAAGGTGACCAAGTTCATGCTATTCCCTTTCACCTTTTGCg & Forward primer \\
TAC1-R & GAAGGTCGGAGTCAACGGATTATTCCCTTTCACCTTTTGCa & Forward primer \\
$\# 1$ & GACATGGATGTTTCTCTTGAGC & Reverse primers \\
\#2 & GAAGGTCGGAGTCAACGGATT-HEX & $\begin{array}{c}\text { Common primer labeled with the FAM } \\
\text { fluorophore }\end{array}$ \\
& & $\begin{array}{c}\text { Common primer labeled with the HEX } \\
\text { fluorophore }\end{array}$ \\
\hline
\end{tabular}

Sequence verification of the SNP variations

The online primer design tool Primer3Plushttps:// primer3plus.com) was utilized to design the primer sets, the forward primer TAC1F1 (5'GGCTGGAGCTATGATGGACC- $3^{\prime}$ ) and the reverse primer TAC1R1 (5'-ATCACTGGCGAGCGAATAGG-3'). PCR products were Sanger-sequenced and the CLUSTAL W soft (https://myhits.sib.swiss/cgibin/clustalw) was used for sequence alignment. The PCR reaction system $(10 \mu \mathrm{L})$ included $1 \mu \mathrm{L}$ $10 \times$ PCR mix (containing PCR buffer, dNTP and Taq enzymes), $0.15 \mu \mathrm{L} 10 \mathrm{mM}$ TAC1F1 primer, 0.15 $\mu \mathrm{L} 10 \mathrm{mM}$ TAC1R1 primer, $1 \mu \mathrm{L} 50$ ng DNA template, and $7.7 \mu \mathrm{L}$ of $\mathrm{ddH}_{2} \mathrm{O}$. PCR was performed as follows: $95{ }^{\circ} \mathrm{C}$ for $5 \mathrm{~min}$, followed by 30 cycles of $95{ }^{\circ} \mathrm{C}$ for $30 \mathrm{~s}$ and $57{ }^{\circ} \mathrm{C}$ for $30 \mathrm{~s}$ and $72{ }^{\circ} \mathrm{C}$ for $30 \mathrm{~s}$, and $72{ }^{\circ} \mathrm{C}$ for $5 \mathrm{~min}$.

\section{Utilization of $t a c l$ in breeding}

The L204 of compact plant architecture was hybridized with the R998 of loose plant architecture. R998 was backcrossed as a recurrent parent with $F_{1}$ generation, and the $\mathrm{BC}_{1} \mathrm{~F}_{1}$ population was obtained. From $\mathrm{BC}_{1} \mathrm{~F}_{1}$ generation, the PM-TAC1 fluorescent molecular marker was used to track and detect each generation. The lines of the tacl allele (G) type and compact plant architecture were selected to backcross with $\mathrm{R} 998$, the $\mathrm{BC}_{2} \mathrm{~F}_{1}$ population was obtained. After two generations of backcross and one generation of selfcross, the $\mathrm{BC}_{2} \mathrm{~F}_{2}$ population was obtained. The breeding material of the tacl allele (G) type and compact plant architecture was selected. Finally,
$\mathrm{BC}_{2} \mathrm{~F}_{3}$ was selected as the breeding material of stable genetic compact plant architecture.

\section{Results}

Development of TAC1 PARMS marker

Ninety-three rice materials were genotyped using the TACl functional molecular fluorescence marker PMTAC1. PM-TAC1 matched well with the corresponding SNP to generate a fluorescence signal and achieve the purpose of genotyping (Table S2). Results showed that 22 materials were $t a c 1$ genotypes, 70 materials were TACl genotypes, and 1 materials was a TAC1/ tacl hybrid genotype (Fig. 1).

The detected FAM fluorescence signal (blue dot) is a compact plant architecture rice variety with tacl genotype. The detected HEX fluorescence signal

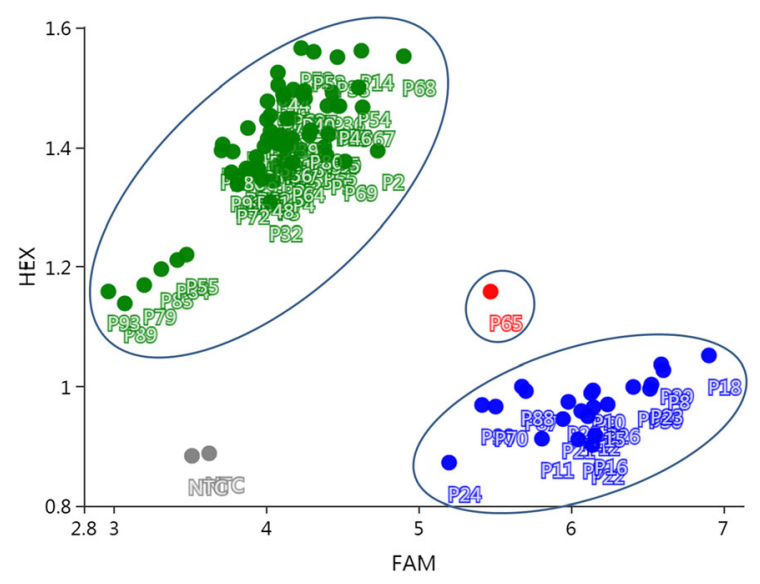

Fig. 1 Genotyping of 93 rice varieties using the molecular marker PM-TAC1 
(green dot) is a loose plant architecture rice variety with TAC1 genotype. The red dot is a heterozygous state. The gray point is negative CK.

Plant architecture identification of rice varieties

The plant architecture was observed and investigated at the heading stage. A compact plant architecture denotes that each tiller is compact, and this type of architecture is found in rice materials LiangfengB, TaifengB, and Gui316 (Fig. 2). A loose plant architecture has a certain angle for each tiller, and this characteristic is observed in Shen08S, GR076, and RP07-1 (Fig. 3). The results of sequence verification by Sanger-sequencing confirmed the SNP variation are correctly corresponding to phenotypes in our material (Fig. 4). LiangfengB, TaifengB, and Gui316, which is compact plant architecture, was the tacl allele (G) type; and Shen08S, GR076, and RP07-1, which is loose plant architecture, was the TACl allele (A) type. The plant architectures of 93 rice materials were investigated (Table 2). Results showed that 23 rice materials had a compact plant architecture, whereas 70 rice materials had a loose plant architecture.
Polymorphism of PM-TAC1 among rice material

The PM-TAC1 fluorescent molecular markers developed herein were used to detect the 93 rice varieties. The amplification results of each rice material were classified according to fluorescent signals (Table S2, Fig. 1). The genotypes of each rice material were classified based on scatter diagram (Table 2). The scatter diagram intuitively classified the corresponding genotype of each material. The green dot represents the sample of HEX fluorescence signal, a loose TAC1 genotype; the blue dot denotes the sample of FAM fluorescence signal, a compact tacl genotype. A comparison of the statistical data revealed that the phenotypic differentiation of the marker PM-TAC1 was consistent with the field phenotypes statistics (Table 2), indicating that the fluorescent molecular marker PM-TAC1 quickly and accurately detected the SNP variations.

\section{Utilization of PM-TAC1 in rice breeding}

Backcross breeding was conducted using L204 (compact plant architecture) and R998 (loose plant architecture) to breed the compact plant architecture material(Fig. 5A). PM-TAC1 was used to detect 378 randomly selected individual plants in the $\mathrm{BC}_{2} \mathrm{~F}_{3}$
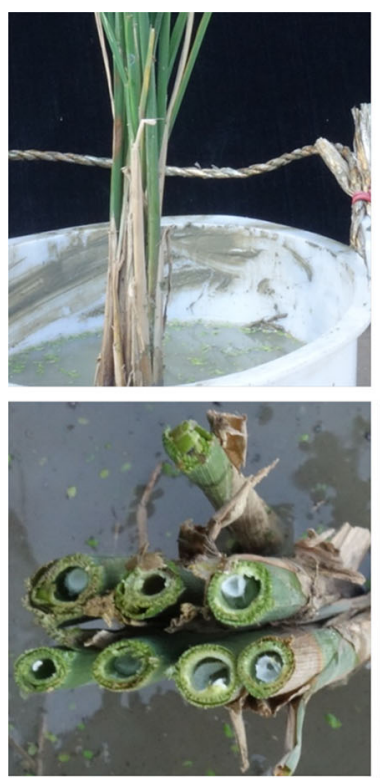

LiangfengB
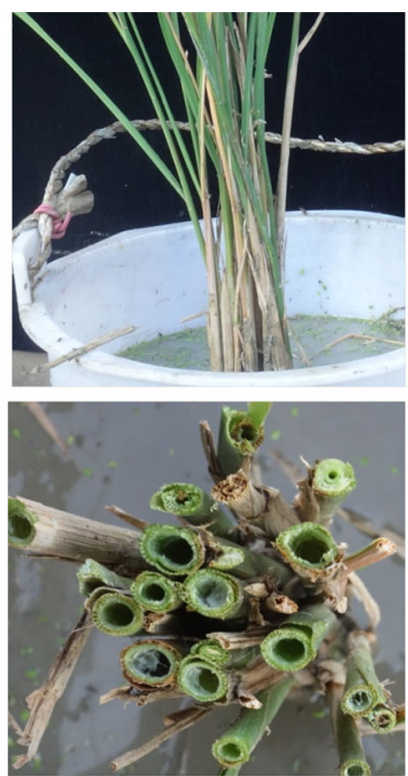

TaifengB
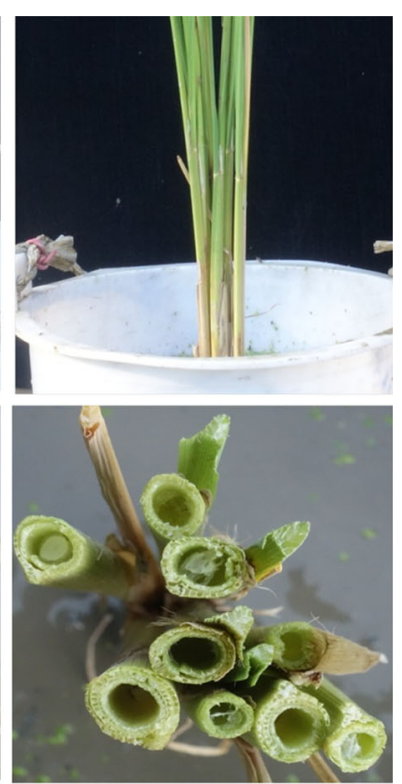

Gui316

Fig. 2 Rice varieties with a compact plant architecture 
Fig. 3 Rice varieties with a loose plant architecture
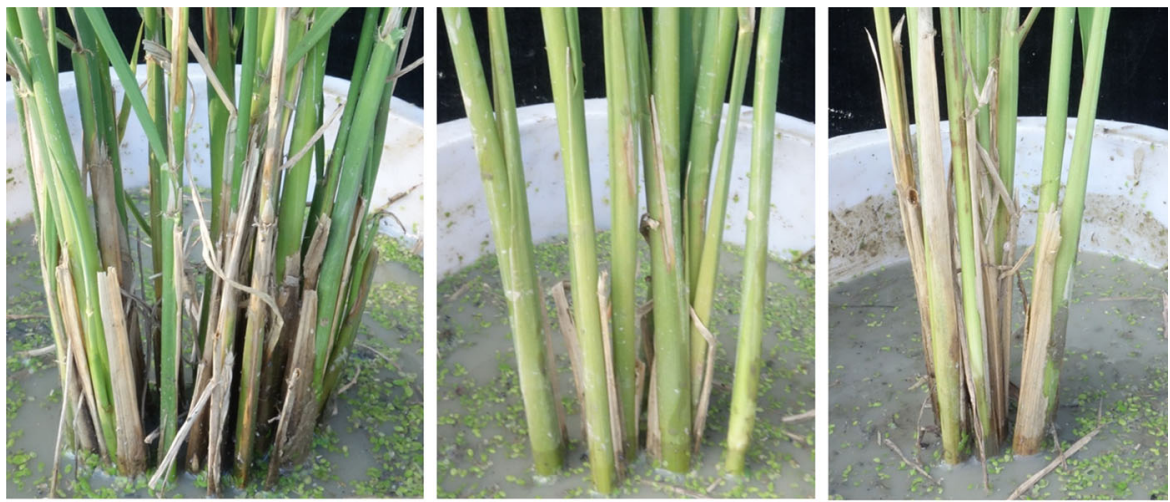

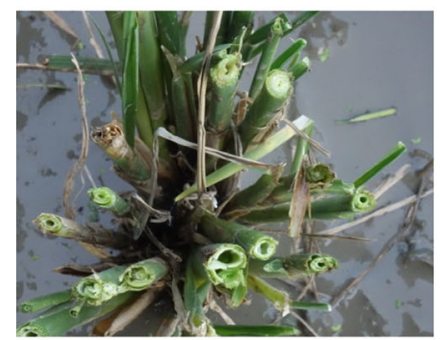

Shen08S

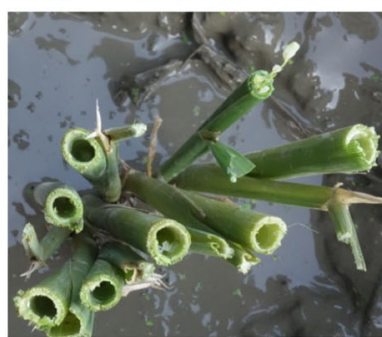

GR076

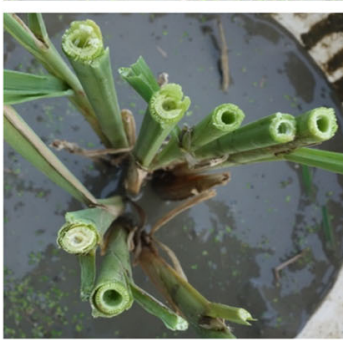

RP07-1 population (Table S3, Fig. 5B). In the scatter diagram, the corresponding genotype of each material is accurately classified to the loose $T A C 1$ genotype (the green dot), the compact tacl genotype (the blue dot) and the TAC1/tacl heterozygous genotype (the red dot). The genotyping is accurately to selecte the target plant architecture (Fig. 5C, Fig. 5D). The results demonstrated that PM-TAC1 can be effectively used in plant architecture molecular breeding of rice.

\section{Discussion}

PM-TAC1 is suitable for plant architecture breeding of rice

The realization of ideal plant architecture is an ideal state of design breeding (Zhang 2007). A large tiller angle increases leaf shade and reduces photosynthesis efficiency. By contrast, a small tiller angle makes the plant structure more effective. A reasonable tillering angle is a key factor for cultivating the ideal plant architecture to achieve high-yield breeding. Highyield rice populations require a loose and moderate plant architecture. An appropriate tiller angle is also conducive to enhancing rice photosynthesis, improving resistance to lodging and sheath blight disease
(Sun et al. 2019), and improving plant yield and rice harvest index. Moreover, tiller angle directly determines the planting density of rice and has a considerable influence on field population yield.

The tiller angle gene, which controls the extremely dispersed or erect types of rice, has little practical breeding value. By contrast, QTLs with certain effects have great value in plant architecture improvement. The major QTL TACl can increase the appropriate tillering angle of rice lines, a characteristic that is conducive to reducing the harm of sheath blight and has no adverse effects on other agronomic traits (Yu et al. 2007; Jiang et al. 2012). The results of the present study demonstrated that $T A C l$ can lead to a reasonable tillering angle and can be used for plant architecture improvement of indica and japonica rice varieties. SNP variations from A to $\mathrm{G}$ at the $3^{\prime}$ junction of intron 4 of $T A C l$ changed the plant architecture of rice from loose to compact (Yu et al. 2007). SNP variations in this locus can be used as a functional molecular marker in rice breeding. Based on these SNP variations, we successfully developed the PARMS marker PMTAC1 of TAC1. This marker has achieved accurate genotyping in 93 rice materials (Fig. 1). The genotypes were consistent with the phenotypic results, and the marker was effectively applied to the plant architecture breeding of indica hybrid rice. The loose 


Shen08S
RP07-1
R998
GR076
L204
LiangfengB
TaifengB
Gui316

Shen08S
RP07-1
R998
GR076
L204
LiangfengB
TaifengB
Gui316
Rhen08S
RP07-1
G998
L204
LiangfengB
Gui316

AAAATTTCTGGCCGACAATACGTCTTGATCAGGAACTGACTAAAGAAATTCCCTTTCACC AAAATTTCTGGCCGACAATACGTCTTGATCAGGAACTGACTAAAGAAATTCCCTTTCACC AAAATTTCTGGCCGACAATACGTCTTGATCAGGAACTGACTAAAGAAATTCCCTTTCACC AAAATTTCTGGCCGACAATACGTCTTGATCAGGAACTGACTAAAGAAATTCCCTTTCACC AAAATTTCTGGCCGACAATACGTCTTGATCAGGAACTGACTAAAGAAATTCCCTTTCACC AAAATTTCTGGCCGACAATACGTCTTGATCAGGAACTGACTAAAGAAATTCCCTTTCACC AAAATTTCTGGCCGACAATACGTCTTGATCAGGAACTGACTAAAGAAATTCCCTTTCACC AAAATTTCTGGCCGACAATACGTCTTGATCAGGAACTGACTAAAGAAATTCCCTTTCACC ******************************************************************

TTTTGCAGGATTTGTTGGATCCATCCTCACGCTTCGGATTCCTTGCTCAAGAGAAACATC TTTTGCAGGATTTGTTGGATCCATCCTCACGCTTCGGATTCCTTGCTCAAGAGAAACATC TTTTGCAGGATTTGTTGGATCCATCCTCACGCTTCGGATTCCTTGCTCAAGAGAAACATC TTTTGCAGGATTTGTTGGATCCATCCTCACGCTTCGGATTCCTTGCTCAAGAGAAACATC TTTTGCGGGATTTGTTGGATCCATCCTCACGCTTCGGATTCCTTGCTCAAGAGAAACATC TTTTGEGGGATTTGTTGGATCCATCCTCACGCTTCGGATTCCTTGCTCAAGAGAAACATC TTTTGCGGGATTTGTTGGATCCATCCTCACGCTTCGGATTCCTTGCTCAAGAGAAACATC TTTTGCGGGATTTGTTGGATCCATCCTCACGCTTCGGATTCCTTGCTCAAGAAAAACATC ****** ********************************************** $* * * * * * *$

CATGCATATCGTCGACAGCGTTCGTTCAGTCCTCTTCCTTTTGTTGTTGTTGCTGTTGTT CATGCATATCGTCGACAGCGTTCGTTCAGTCCTCTTCCTTTTGTTGTTGTTGCTGTTGTT CATGCATATCGTCGACAGCGTTCGTTCAGTCCTCTTCCTTTTGTTGTTGTTGCTGTTGTT CATGCATATCGTCGACAGCGTTCGTTCAGTCCTCTTCCTTTTGTTGTTGTTGCTGTTGTT CATGCATATCGTCGACAGCGTTCGTTCAGTCCTCTTCCTTTTGTTGTTGGTGCTGTTGTT CATGCATATCGTCGACAGCGTTCGTTCAGTCCTCTTCCTTTTGTTGTTGGTGCTGTTGTT CATGCATATCGTCGACAGCGTTCGTTCAGTCCTCTTCCTTTTGTTGTTGGTGCTGTTGTT CATGCATATCGTCGACAGCGTTCGTTCAGTCCTCTTCCTTTTGTTGTTGGGGCTGTTGTT ************************************************* $\quad * * * * * * * * *$

Fig. 4 Sequence verification of the SNP variations. The SNP variation in the box is exploited by the PM-TAC1 markers

plant architecture of the rice variety L204 was improved and an L204 material with compact plant architecture was obtained via molecular marker assisted breeding (Fig. 5).

PM-TAC1 based on PARMS is a highly efficient marker for MAS breeding

The development of functional molecular markers of target trait genes is an important aspect of directional improvement breeding( $\mathrm{Xu}$ and Crouch 2008). As the most potential marker of the third-generation molecular markers, SNP markers have developed rapidly and been widely used in genetic analysis. Gel electrophoresis-based molecular markers such as single-strand conformational polymorphism (SSCP), denaturing gradient gel electrophoresis (DDGE), cleaved amplified polymorphic sequence (CAPS), and allele-specific PCR (AS-PCR) is limited because electrophoresis is laborious, time-consuming, requires 
Table 2 PM-TAC1 marker genotypes and plant architecture phenotypes of 93 rice varieties

\begin{tabular}{|c|c|c|c|c|c|c|c|}
\hline No & Variety & Genotype & Phenotype & No & Variety & Genotype & Phenotype \\
\hline P1 & MeiB & $T A C 1$ & Loose & P48 & Lixiang2 & $T A C 1$ & Loose \\
\hline $\mathrm{P} 2$ & $82 \mathrm{~B}$ & $T A C 1$ & Loose & P49 & R308 & $T A C 1$ & Loose \\
\hline $\mathrm{P} 3$ & $112 B$ & $T A C 1$ & Loose & P50 & R318 & $T A C 1$ & Loose \\
\hline P4 & 186B & $T A C 1$ & Loose & P51 & R319 & $T A C 1$ & Loose \\
\hline P5 & 9311B & $T A C 1$ & Loose & P52 & $\mathrm{R} 402$ & $T A C 1$ & Loose \\
\hline P6 & II-32B & tacl & Compact & P53 & R463 & $T A C 1$ & Loose \\
\hline P7 & II-33B(an) & tacl & Compact & P54 & R527 & $T A C 1$ & Loose \\
\hline P8 & BoB & tacl & Compact & P55 & IRBB5 & $T A C 1$ & Loose \\
\hline P9 & BoIIB & tacl & Compact & P56 & R5867 & TAC1 & Loose \\
\hline P10 & FuyiB & tacl & Compact & P57 & R7571 & $T A C 1$ & Loose \\
\hline P11 & Gang46B & tacl & Compact & P58 & R774 & $T A C 1$ & Loose \\
\hline P12 & GufengB & tacl & Compact & P59 & R789 & $T A C 1$ & Loose \\
\hline P13 & Guang8B & $T A C 1$ & Loose & P60 & R795 & $T A C 1$ & Loose \\
\hline P14 & HenfengB & $T A C 1$ & Loose & P61 & R9516 & $T A C 1$ & Loose \\
\hline P15 & Hua2048B & tacl & Compact & P62 & R087 & $T A C 1$ & Loose \\
\hline P16 & Jin23B & tacl & Compact & P63 & R087 & TAC1 & Loose \\
\hline P17 & LiangfengB & tacl & Compact & P64 & RP07-1 & $T A C 1$ & Loose \\
\hline P18 & ShanB & tacl & Compact & P65 & Bing4114 & TAC1/tacl & Loose \\
\hline P19 & Shen95B & $T A C 1$ & Loose & P66 & $\mathrm{Ce} 253$ & $T A C 1$ & Loose \\
\hline $\mathrm{P} 20$ & TaifengB & tacl & Compact & P67 & Duoxi1 & $T A C 1$ & Loose \\
\hline $\mathrm{P} 21$ & $\mathrm{TeB}$ & tacl & Compact & P68 & R998 & $T A C 1$ & Loose \\
\hline P22 & TianfengB & tacl & Compact & P69 & Gui3158 & TAC1 & Loose \\
\hline $\mathrm{P} 23$ & WufengB & tacl & Compact & P70 & Gui316 & tacl & Compact \\
\hline P24 & BoIIB & tacl & Compact & P71 & Gui501 & TAC1 & Loose \\
\hline $\mathrm{P} 25$ & YexiangB & TAC1 & Loose & P72 & Gui826 & TAC1 & Loose \\
\hline $\mathrm{P} 26$ & YixiangB & tacl & Compact & P73 & Gui965 & $T A C 1$ & Loose \\
\hline $\mathrm{P} 27$ & Yu19B & $T A C 1$ & Loose & P74 & Gui99 & TAC1 & Loose \\
\hline P28 & Yue4B & $T A C 1$ & Loose & P75 & GuiR100 & $T A C 1$ & Loose \\
\hline P29 & ZhenfengB & tacl & Compact & P76 & Huazhan & TAC1 & Loose \\
\hline P30 & ZhongjiuB & tacl & Compact & P77 & Minghui63 & TAC1 & Loose \\
\hline P31 & ZhongzheB & $T A C 1$ & Loose & P78 & Yahui2155 & TAC1 & Loose \\
\hline P32 & LongS & $T A C 1$ & Loose & P79 & Guike1 & TAC1 & Loose \\
\hline P33 & JingS & $T A C 1$ & Loose & P80 & Guiyu9 & TAC1 & Loose \\
\hline P34 & Y58S & $T A C 1$ & Loose & P81 & Wushansimiao & TAC1 & Loose \\
\hline P35 & Shen08S & $T A C 1$ & Loose & P82 & 2428 & $T A C 1$ & Loose \\
\hline P36 & He620S & $T A C 1$ & Loose & P83 & Nante & TAC1 & Loose \\
\hline P37 & $1892 \mathrm{~S}$ & $T A C 1$ & Loose & P84 & Zhenguiai & $T A C 1$ & Loose \\
\hline P38 & 9311 & $T A C 1$ & Loose & P85 & Gumei4 & $T A C 1$ & Loose \\
\hline P39 & $838 \mathrm{R}(\mathrm{L})$ & $T A C 1$ & Loose & P86 & ВР60 & $T A C 1$ & Loose \\
\hline P40 & Fuhui838 & $T A C 1$ & Loose & P87 & Jinweiai & tacl & Compact \\
\hline P41 & GR076 & TAC1 & Loose & P88 & Kongyu131 & tacl & Compact \\
\hline $\mathrm{P} 42$ & GS2 & TAC1 & Loose & P89 & Dayu23 & TAC1 & Loose \\
\hline $\mathrm{P} 43$ & IRBB7 & TAC1 & Loose & P90 & L204 & tacl & Compact \\
\hline P44 & $\mathrm{R} 128$ & $T A C 1$ & Loose & P91 & LCL & $T A C 1$ & Loose \\
\hline $\mathrm{P} 45$ & R138 & $T A C 1$ & Loose & P92 & Hongnanzao & TAC1 & Loose \\
\hline
\end{tabular}


Table 2 continued

\begin{tabular}{llllllll}
\hline No & Variety & Genotype & Phenotype & No & Variety & Genotype & Phenotype \\
\hline P46 & R1439 & TAC1 & Loose & P93 & Honggui6 & TAC1 \\
P47 & R273 & TAC1 & Loose & & & & Loose \\
\hline
\end{tabular}

A

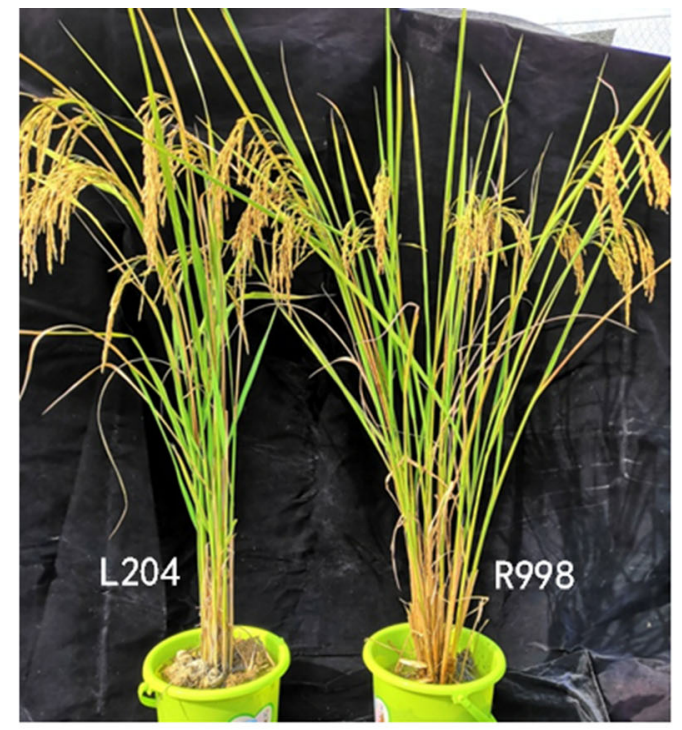

C

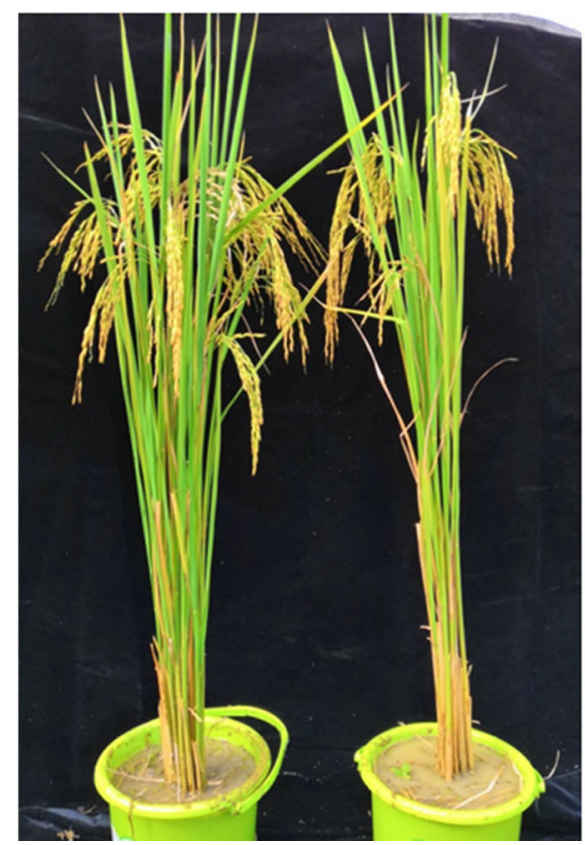

Fig. 5 PM-TAC1 genotypes and plant architecture phenotypes of the $\mathrm{BC}_{2} \mathrm{~F}_{3}$ breeding population. A. plant architecture of the two parents L204 and R998. B. PM-TAC1 genotyping of 378 randomly selected individual plants in the $\mathrm{BC}_{2} \mathrm{~F}_{3}$ population. $\mathbf{C}$.

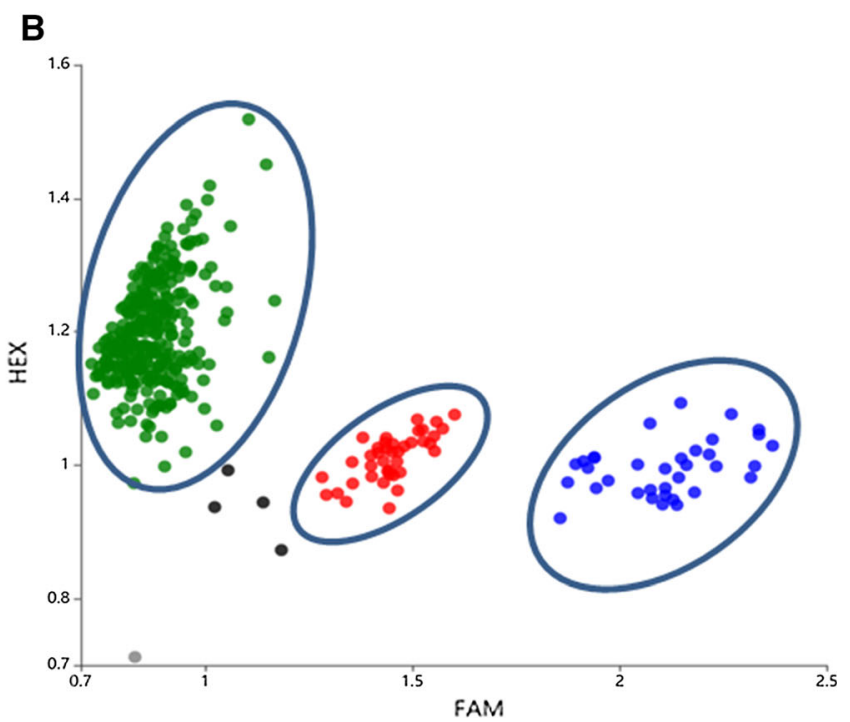

D

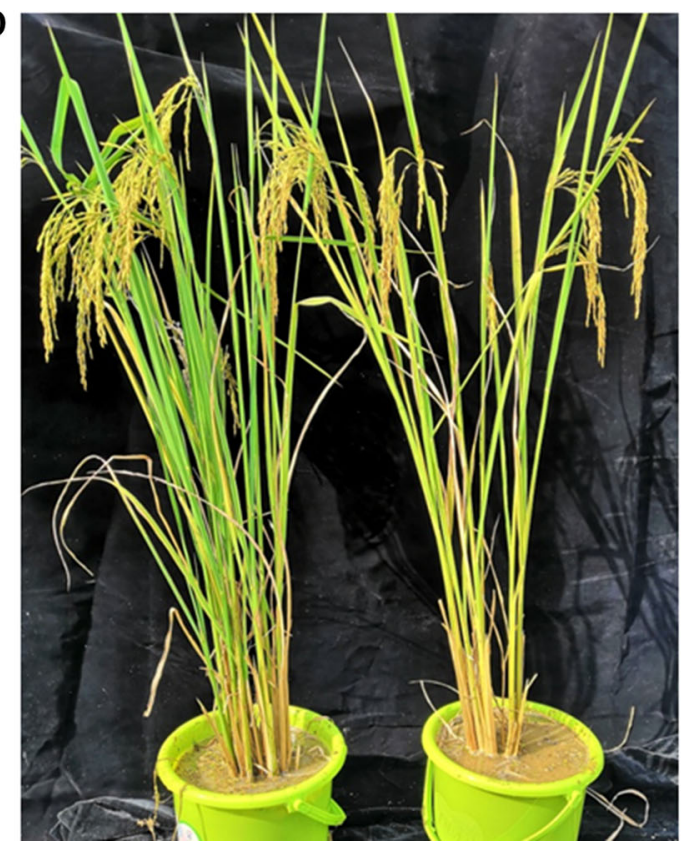

compact plant architecture of tacl genotype in the $\mathrm{BC}_{2} \mathrm{~F}_{3}$ population. D. loose plant architecture of TACl genotype in the $\mathrm{BC}_{2} \mathrm{~F}_{3}$ population. 
toxic reagents, and complicated. The gel-free SNP marker system such as direct sequencing, DNA chips, denaturing high-performance liquid chromatography (DHPLC), mass spectrometry detection technology, and high resolution melting (HRM) is high throughput and automated detection methods, which is too expensive to afford for most breeders (Thomson 2014; Batley 2015). Compared with these methods, PARMS based on fluorescence detection has the advantages of simple operation, short operation period, and low cost. Moreover, this technique can detect SNPs and short indel loci (Zhang et al. 2019a, b; Lu et al. 2020). In PARMS, two different universal primers are added to the end of the primers. With the participation of universal fluorescent primers in the amplification system, different genotypes are amplified to obtain different fluorescent groups. The detection samples are amplified by PCR only once without electrophoresis detection, and the amplification data are directly obtained from the original plate by a fluorescent scanner. After software analysis, the amplification data and the TACl genotypic data are obtained. Our results demonstrated that PM-TAC1 by PARMS is a highly efficient marker for MAS breeding (Fig. 5).

\section{Conclusion}

In this study, the TACl fluorescence marker PMTAC1 was developed via the PARMS technology. This fluorescence marker can be detected at any stage of rice growth. It is simple, fast, inexpensive, and can obtain accurate results. Moreover, this marker can realize closed tube operation. This functional marker of the rice tiller angle gene $T A C l$ was successfully developed and effectively used in breeding new plant architecture breeding of rice.

Acknowledgements The authors thank Fei Chen and Jiali Liu for assistance in the field experiments and data collection.

Author contributions JG, HFL, LJG, and GFD: conceived the original plan and designed the research; JG, HFL and LJG: performed the experiments and collected the data; JG: wrote the manuscript; JH, DJQ, HW, WYZ, WWC, YHP and GXD: performed manuscript modification; GFD: supervised the project and overviewed all aspects of the work.

Funding This work was financially supported by funds from The National Key Research and Development Program of China
(2017YFD0100103), Nanning Comprehensive Test Station of National Rice Industry Technology System(CARS-01-88), Special Fund for Guangxi Science and Technology Base and Talents (GuiKe AD17129064), Guangxi Natural Science Foundation (2018GXNSFAA281202), Guangxi Science and Technology Base and Special Talents (GuiKe AD18050003), Special Fund for Basic Scientific Research of Guangxi Academy of Agricultural Sciences (GuiNongKe2020YM06).

\section{Compliance with ethical standards}

Conflict of interest The authors declare that they have no conflict of interest.

Ethical approval The experiments comply with the current laws of the country in which they were performed.

Open Access This article is licensed under a Creative Commons Attribution 4.0 International License, which permits use, sharing, adaptation, distribution and reproduction in any medium or format, as long as you give appropriate credit to the original author(s) and the source, provide a link to the Creative Commons licence, and indicate if changes were made. The images or other third party material in this article are included in the article's Creative Commons licence, unless indicated otherwise in a credit line to the material. If material is not included in the article's Creative Commons licence and your intended use is not permitted by statutory regulation or exceeds the permitted use, you will need to obtain permission directly from the copyright holder. To view a copy of this licence, visit http://creativecommons.org/licenses/by/4.0/.

\section{References}

Batley J (ed) (2015) Plant genotyping: methods and protocols. Springer, New York

Chen Y, Fan X, Song W, Zhang Y, Xu G (2012) Over-expression of OsPIN2 leads to increased tiller numbers, angle and shorter plant height through suppression of OsLAZY1. Plant Biotechnol J 10(2):139-149. https://doi.org/10.1111/ j.1467-7652.2011.00637.x

Dong H, Zhao H, Xie W, Han Z, Li G, Yao W, Bai X, Hu Y, Guo Z, Lu K, Yang L, Xing Y (2016) A novel tiller angle gene, TAC3, together with TAC1 and D2 largely determine the natural variation of tiller angle in rice cultivars. Plos Genet 12(11):e1006412. https://doi.org/10.1371/journal.pgen. 1006412

Jiang J, Tan L, Zhu Z, Fu Y, Liu F, Cai H, Sun C (2012) Molecular evolution of the TAC1 gene from rice (Oryza sativa L.). J Genet Genomics 39(10):551-560. https://doi. org/10.1016/j.jgg.2012.07.011

Jin J, Huang W, Gao JP, Yang J, Shi M, Zhu MZ, Luo D, Lin HX (2008) Genetic control of rice plant architecture under domestication. Nat Genet 40(11):1365-1369. https://doi. org/10.1038/ng.247

Khush G (1996) Prospects of and approaches to increasing the genetic yield potential of rice. In: Evenson EA (ed) Rice 
research in Asia, progress and priorities. $\mathrm{CAB}$ international and IRRI, Wallingford, pp 59-71

Li P, Wang Y, Qian Q, Fu Z, Wang M, Zeng D, Li B, Wang X, Li J (2007) LAZY1 controls rice shoot gravitropism through regulating polar auxin transport. Cell Res 17(5):402-410. https://doi.org/10.1038/cr.2007.38

Li Z, Liang Y, Yuan Y, Wang L, Meng X, Xiong G, Zhou J, Cai Y, Han N, Hua L, Liu G, Li J, Wang Y (2019) OsBRXL4 regulates shoot gravitropism and rice tiller angle through affecting LAZY1 nuclear localization. Mol Plant 12(8):1143-1156. https://doi.org/10.1016/j.molp.2019.05. 014

Li Y, Li J, Chen Z, Wei Y, Qi Y, Wu C (2020) OsmiR167atargeted auxin response factors modulate tiller angle via fine-tuning auxin distribution in rice. Plant Biotechnol J. https://doi.org/10.1111/pbi.13360

Liu JM, Park SJ, Huang J, Lee EJ, Xuan YH, Je BI, Kumar V, Priatama RA, Raj KV, Kim SH, Min MK, Cho JH, Kim TH, Chandran AK, Jung KH, Takatsuto S, Fujioka S, Han CD (2016) Loose Plant Architecture1 (LPA1) determines lamina joint bending by suppressing auxin signalling that interacts with C-22-hydroxylated and 6-deoxo brassinosteroids in rice. J Exp Bot 67(6):1883-1895. https://doi.org/ 10.1093/jxb/erw002

Lu J, Hou J, Ouyang Y, Luo H, Zhao J, Mao C, Han M, Wang L, Xiao J, Yang Y, Li X (2020) A direct PCR-based SNP marker-assisted selection system (D-MAS) for different crops. Mol Breeding 40(1):9. https://doi.org/10.1007/ s11032-019-1091-3

Miyata M, Komori T, Yamamoto T, Ueda T, Yano M, Nitta N (2005) Fine Scale and Physical Mapping of Spk(t) Controlling Spreading Stub in Rice. Breeding Sci 55(2):237-239. https://doi.org/10.1270/jsbbs.55.237

Murra y MG, Thompson WF (1980) Rapid isolation of high molecular weight plant DNA. Nucleic Acids Res 8(19):4321-4325. https://doi.org/10.1093/nar/8.19.4321

Sun Q, Li TY, Li DD, Wang ZY, Li S, Li DP, Han X, Liu JM, Xuan YH (2019) Over expression ofLoose Plant Architecture 1 increases planting density and resistance to sheath blight disease via activation of PIN-FORMED 1a in rice. Plant Biotechnol J 17(5):855-857. https://doi.org/10.1111/ pbi. 13072

Tan L, Li X, Liu F, Sun X, Li C, Zhu Z, Fu Y, Cai H, Wang X, Xie D, Sun C (2008) Control of a key transition from prostrate to erect growth in rice domestication. Nat Genet 40(11):1360-1364. https://doi.org/10.1038/ng.197

Thomson MJ (2014) High-throughput snp genotyping to accelerate crop improvement. Plant Breed Biotechnol 2(3):195-212. https://doi.org/10.9787/PBB.2014.2.3.195

Wang Y, Li J (2005) The plant architecture of rice (Oryza sativa). Plant Mol Biol 59(1):75-84. https://doi.org/10. 1007/s11103-004-4038-x

Wang L, Xu Y, Zhang C, Ma Q, Joo SH, Kim SK, Xu Z, Chong K (2008) OsLIC, a novel CCCH-type zinc finger protein with transcription activation, mediates rice architecture via brassinosteroids signaling. PLoS ONE 3(10):e3521. https://doi.org/10.1371/journal.pone.0003521

Wu X, Tang D, Li M, Wang K, Cheng Z (2013) Loose Plant Architecture1, an INDETERMINATE DOMAIN protein involved in shoot gravitropism, regulates plant architecture in rice. Plant Physiol 161(1):317-329. https://doi.org/10. 1104/pp.112.208496

Xie C, Zhang G, An L, Chen X, Fang R (2019) Phytochromeinteracting factor-like protein OsPIL15 integrates light and gravitropism to regulate tiller angle in rice. Planta 250(1):105-114. https://doi.org/10.1007/s00425-01903149-8

Xu Y, Crouch JH (2008) Marker-assisted selection in plant breeding: from publications to practice. Crop Sci 48(2):391-407. https://doi.org/10.2135/cropsci2007.04. 0191

Yamamoto T (1997) Genetic analysis of spreading stub using indica/japonica backcrossed progenies in rice. Breeding Sci 47(2):141-144

Ye S, Dhillon S, Ke X, Collins AR, Day IN (2001) An efficient procedure for genotyping single nucleotide polymorphisms. Nucl Acids Res 29(17):E88. https://doi.org/10. 1093/nar/29.17.e88

Yu B, Lin Z, Li H, Li X, Li J, Wang Y, Zhang X, Zhu Z, Zhai W, Wang X, Xie D, Sun C (2007) TAC1, a major quantitative trait locus controlling tiller angle in rice. Plant $\mathrm{J}$ 52(5):891-898. https://doi.org/10.1111/j.1365-313X. 2007.03284.x

Zhang Q (2007) Strategies for developing green super rice. Proc Natl Acad Sci 104(42):16402-16409. https://doi.org/10. 1073/pnas.0708013104

Zhang C, Xu Y, Guo S, Zhu J, Huan Q, Liu H, Wang L, Luo G, Wang X, Chong K (2012) Dynamics of brassinosteroid response modulated by negative regulator LIC in rice. Plos Genet 8(4):e1002686. https://doi.org/10.1371/journal. pgen. 1002686

Zhang W, Tan L, Sun H, Zhao X, Liu F, Cai H, Fu Y, Sun X, Gu P, Zhu Z, Sun C (2019) Natural variations at TIG1 encoding a TCP transcription factor contribute to plant architecture domestication in rice. Mol Plant 12(8):1075-1089. https://doi.org/10.1016/j.molp.2019.04. 005

Zhang B, Zhao N, Liu Y, Jia L, Fu Y, He X, Liu K, Xu Z, Bao B (2019) Novel molecular markers for high-throughput sex characterization of Cynoglossus semilaevis. Aquaculture 513:734331. https://doi.org/10.1016/j.aquaculture.2019. 734331

Publisher's Note Springer Nature remains neutral with regard to jurisdictional claims in published maps and institutional affiliations. 